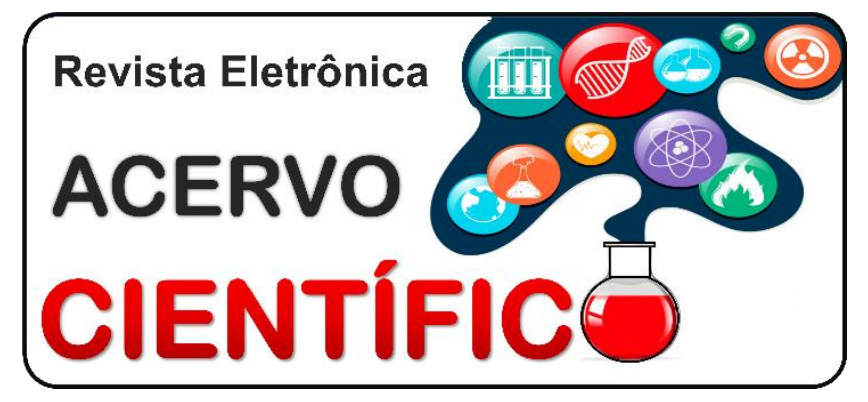

\section{REVISÃO BIBLIOGRÁFICA}

Recebido em: 5/2020

Aceito em: 6/2020

Publicado em: 9/2020

\title{
O uso da hidroterapia como recurso na melhora da espasticidade muscular em pacientes com sequelas do avc: uma revisão sistemática
}

\author{
The use of hydrotherapy as a resource in improving muscular spasticity in patients with \\ stroke sequels: a systematic review
El uso de la hidroterapia como recurso para mejorar la espasticidad muscular en pacientes con secuelas de la estructura: una revisión sistemática

Pamela Beatriz Caresto Batista ${ }^{1 *}$, Geisiandre Barbosa dos Santos ${ }^{1}$, Ejandre Garcia Negreiros da Silva $^{2}$, Joanne Figueiredo de Oliveira ${ }^{1}$.

\begin{abstract}
Resumo: O objetivo do presente estudo foi analisar através de uma revisão sistemática a respeito dos efeitos da hidroterapia na diminuição da espasticidade muscular resultando na melhora da marcha de pacientes com sequelas de Acidente Vascular Cerebral (AVC). Foi realizada uma revisão de literatura a partir da coleta de estudos disponíveis nas bibliotecas virtuais PubMED, SciELO e MEDLINE no período de março a abril de 2020, onde foram selecionados os artigos cujo o tema e resumo se apresentavam de acordo com a proposta do trabalho e as revisões foram avaliadas pela escala Revised Assessment of Multiple Systematic Reviews (R-AMSTAR). Foram encontrados 156 estudos, no entanto, 152 foram excluídos, restando apenas 4 estudos que se enquadravam nos critérios de inclusão e exclusão para inclusão nesta revisão. Concluímos que a hidroterapia oferece resultados satisfatórios quando combinada com outros métodos de tratamento, é um recurso eficaz na melhora do equilíbrio, no ganho de força muscular e na diminuição da espasticidade muscular.
\end{abstract}

Palavras-chave: Hidroterapia, Fisioterapia, Acidente Vascular Cerebral.

\begin{abstract}
The objective of the present study was to analyze through a systematic review regarding the effects of hydrotherapy in reducing muscle spasticity resulting in improved gait in patients with stroke sequelae. A literature review was carried out based on the collection of studies available in the virtual libraries PubMED, SciELO and MEDLINE from March to April 2020, where articles were selected whose theme and summary were in accordance with the work proposal and revisions were assessed using the Revised Assessment of Multiple Systematic Reviews (R-AMSTAR) scale. 156 studies were found, however, 152 were excluded, leaving only 4 studies that met the inclusion and exclusion criteria for inclusion in this review. We conclude that hydrotherapy offers satisfactory results when combined with other treatment methods, it is an effective resource in improving balance, gaining muscle strength and decreasing muscle spasticity.
\end{abstract}

Keywords: Hydrotherapy, Physiotherapy, Stroke.

${ }^{1}$ Centro Universitário do Norte (UNINORTE), Manaus - AM. *E-mail: ejandregnsilva@gmail.com

2 Universidade Federal do Amazonas (UFAM), Manaus - AM. 
Resumen: El objetivo del presente estudio fue analizar a través de una revisión sistemática sobre los efectos de la hidroterapia en la reducción de la espasticidad muscular que resulta en una mejor marcha en pacientes con secuelas de accidente cerebrovascular. Se realizó una revisión de la literatura basada en la colección de estudios disponibles en las bibliotecas virtuales PubMED, SciELO y MEDLINE de marzo a abril de 2020, donde se seleccionaron artículos cuyo tema y resumen se presentaron de acuerdo con la propuesta de trabajo y Las revisiones se evaluaron utilizando la escala Revised Assessment of Multiple Systematic Reviews (R-AMSTAR). Se encontraron 156 estudios, sin embargo, 152 fueron excluidos, dejando solo 4 estudios que cumplieron con los criterios de inclusión y exclusión para su inclusión en esta revisión. Concluimos que la hidroterapia ofrece resultados satisfactorios cuando se combina con otros métodos de tratamiento, es un recurso efectivo para mejorar el equilibrio, ganar fuerza muscular y disminuir la espasticidad muscular.

Palabras clave: Hidroterapia, Fisioterapia, Accidente vascular cerebral.

\section{INTRODUÇÃO}

O acidente vascular cerebral (AVC), é caracterizado pela uma interrupção do fluxo sanguíneo de uma ou mais áreas do encéfalo, fazendo com que haja uma diminuição do suprimento de oxigênio, manifestando desenvolvimento acelerado de sinais clínicos de permutação focal ou global. Pode ocorrer de duas formas, por obstrução de uma artéria caracterizando o AVC isquêmico, ou por ruptura de uma artéria caracterizando AVC hemorrágico (SPRINGHOUSE ACC, 2004; ROWLAND, et al., 2002).

A Pesquisa Nacional de Saúde (PNS) de 2013, revelou que cerca de 1,5\% da população brasileira acima dos 18 anos de idade possuem diagnóstico de AVC. A doença é mais comum na população idosa com idade acima dos 56 anos e com maior prevalência em pessoas do sexo masculino. O AVC é um dos principais responsáveis por internações e óbitos, quando ocorre do paciente não vir a óbito, a doença pode resultar em algumas sequelas, afetando vários domínios de sua funcionalidade (BENSENOR IM, et al., 2013; LOCATELLI MC, et al., 2017).

Os sintomas e características podem ser únicos ou combinados a fraqueza muscular, parestesia, hemiparesias, hemiplegias, alterações na fala, na visão, na compreensão, na consciência, na coordenação, na marcha, e além disso, podendo ocorrer uma desordem motora, que se caracteriza pela excitação extrema do reflexo do estiramento com exacerbação dos reflexos profundos e aumento do tônus muscular, chamada espasticidade. Dentre os principais fatores de risco que podem acarretar essa síndrome neurológica, estão: a hipertensão arterial sistêmica (HAS), doenças cardiovasculares, obesidade, tabagismo, etilismo e sedentarismo (PIASSAROLI CAP, et al., 2012; LIANZA S, et al., 2001).

Em relação ao diagnóstico clínico é realizado através da anamnese, exame físico e estudo de neuroimagem, sendo: tomografia computadorizada de crânio, angiografia, antirressonância, angiotomografia, ou exames complementares de urgência: eletrocardiograma (ECG), exames laboratoriais como hemograma e glicemia (BRASIL, 2020).

De acordo com O' Sullivan SB, et al. (2010), umas das formas de tratamento para a população vítima de AVC é a fisioterapia, que tem como objetivos reabilitar e prevenir deformidades e complicações, recuperar funções cerebrais comprometidas pelo AVC, temporárias ou permanentes, reintegrando o paciente à suas atividades diárias, promovendo assim, melhor qualidade de vida e independência, utilizando técnicas da hidroterapia, visto que a imersão aquática possui efeitos fisiológicos consideráveis sobre a homeostase, podendo haver resultados imediatos ou tardios, repercutindo de forma benéfica no tratamento da espasticidade, pois a água aquecida reduz esse sintoma, facilitando o movimento e mobilidade funcional, trazendo ao paciente melhora em sua qualidade de vida (BRUCE E, BECKER AJC, 200; GIMENES RO, et al., 2019; MIRANDA MR, et al., 2018).

A hidroterapia é um dos recursos definidos como o uso externo da água com propósitos terapêuticos a partir da incapacidade funcional que o paciente venham a desenvolver é suma importância que ele realize 
tratamento fisioterapêutico para que os déficits não progridam, vale ressaltar que a hidroterapia utiliza as propriedades físicas da água, com as bases biomecânicas e termodinâmicas da água possibilitando ao paciente alcançar facilmente metas e desafios que por sua vez em solo seriam impossíveis de serem realizados, sendo a hidrostática, hidrodinâmica e termodinâmica seu principal fundamento. Porque as forças físicas da água agem sobre o organismo imerso, ocasionando alterações fisiológicas extensas, afetando quase todos os sistemas do organismo do paciente. As propriedades físicas da água relevantes sobre o corpo imerso são: densidade, empuxo, pressão hidrostática, turbulência, viscosidade, tensão superficial e refração, (FINHOLDT, 2007; ROUTI RG, MORRIS DM, 1997).

Estes efeitos físicos da água são mecânicos e térmicos, e combinados aos efeitos do exercício em imersão resulta em respostas fisiológicas. Os efeitos térmicos da água facilitam a troca de calor com o corpo imerso por duas vias: condução e convecção. A primeira acontece pelo movimento normal de energia do corpo mais quente em direção ao mais frio, já segunda consiste na perda que ocorre pelo movimento da água contra o corpo, mesmo em temperaturas iguais. Os efeitos variam com a duração e tipo do exercício, progressão e intensidade, temperatura da água, postura e patologia do paciente (CHÃO CC, 2004).

Sendo o princípio da flutuação chamado por empuxo, trata-se de uma força de sentido contrário à gravidade, facilitando ao corpo imerso um efeito de sustentação, auxiliando na redução de edemas e facilitando a circulação, favorecendo a redução do peso de suporte. A densidade se trata da combinação entre a massa e o volume do corpo imerso, estipulando a capacidade que o corpo possui para flutuar e submergir ao comparar a densidade com a da água, sendo densidade da água igual a 1, e a de um corpo humano igual de 0,93 , por isso ele submerge. No organismo humano, a densidade varia com a composição corporal, ocasionando que pessoas com maior quantidade de tecido adiposo flutuem com maior facilidade (CHÃO CC, 2004).

O presente trabalho torna-se relevante em razão de que os conhecimentos e as ferramentas disponíveis nem sempre estão adequados para solucionar problemáticas da saúde, por esse motivo faz-se necessário buscar através de estudos, novos protocolos de tratamento que se adequem as possíveis necessidades, visando a evolução e o bem estar desse paciente, com a finalidade de acelerar o processo de reabilitação. Portanto, este estudo tem como objetivo analisar através de uma revisão sistemática a respeito dos efeitos da hidroterapia na diminuição da espasticidade resultando na melhora da marcha de pacientes com sequelas de AVC.

\section{MÉTODOS}

Foi realizada uma revisão sistemática a partir da coleta de estudos disponíveis nas bibliotecas virtuais National Library of Medicine (PubMED), Scientific Electronic Library Online (SciELO) e Medical Literature Analysis and Retrieval System Online (MEDLINE) no período de março a abril de 2020. No qual selecionamos estudos publicados no período de 2010 a 2020, onde utilizamos as seguintes palavraschaves em inglês: Hydrotherapy, stroke, spasticity, e seus correlatos específicos identificados no Medical Subject Headings (MESH) e em português nos Descritores em Ciências da Saúde (DeCs): Hidroterapia, AVC, espasticidade.

Foi selecionado os artigos (Figura 1), cujo o tema e resumo se apresentavam de acordo com a proposta do trabalho e nos critérios de inclusão: sendo ensaios clínicos randomizados e não randomizados, estudos realizados com pacientes de 25 a 65 anos de idade e exclusão: data de publicação, duplicados, artigos incompletos estudos pilotos, revisões de literatura. 
Figura 1 - Fluxograma do processo de inclusão e exclusão.

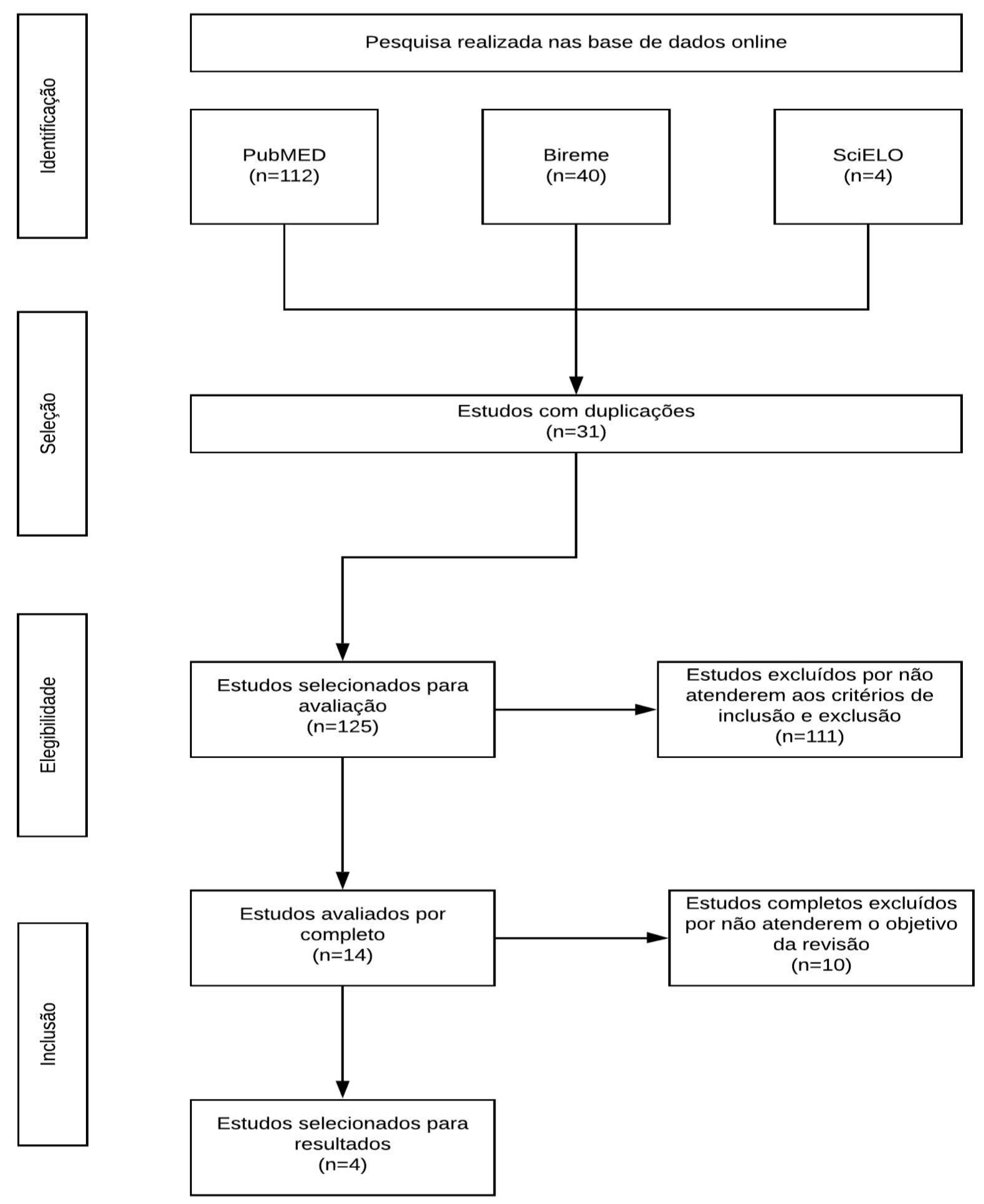

Fonte: Batista PBC, et al., 2020.

As revisões sistemáticas possuem elevado nível de evidência por demonstrar o resultado de vários estudos realizados que abordam um mesmo assunto ou tema e avaliar de forma imparcial a qualidade de cada artigo. Tem uma abordagem metodológica sistematizada de determinado tema proporcionando uma compreensão completa do fenômeno analisado. Diante da necessidade de assegurar uma prática assistencial embasada em evidências científicas, a revisão sistemática tem sido apontada como uma 
ferramenta ímpar no campo da saúde, pois sintetiza as pesquisas disponíveis sobre determinada temática e direciona a prática fundamentando-se em conhecimento científico (DONATO H, DONATO M, 2019).

Somente os artigos em formato de revisão sistemática incluídos neste estudo tiveram a sua qualidade metodológica avaliada através do checklist da Revised Assessment of Multiple Systematic Reviews (RAMSTAR) (Tabela 1), pois é uma ferramenta exclusiva para este tipo de estudo. O R-AMSTAR é uma ferramenta que utiliza 11 perguntas de 4 a 0 pontos cada com score total de 44 pontos. Cada item corresponde a uma pergunta com critérios (sub perguntas) de avaliação: 1) Foi fornecido um design 'a priori'? 3 critérios; 2) Houve seleção duplicada de estudos e extração de dados? 3 critérios; 3) Foi realizada uma pesquisa bibliográfica abrangente? 5 critérios; 4) O status da publicação (literatura cinza) foi usado como critério de inclusão? 4 critérios; 5) Foi fornecida uma lista de estudos (incluídos e excluídos)? 4 critérios; 6) As características dos estudos incluídos foram fornecidas? 3 critérios; 7) A qualidade científica dos estudos incluídos foi avaliada e documentada? 4 critérios; 8) A qualidade científica dos estudos incluídos foi usada adequadamente na formulação de conclusões? 4 critérios; 9) Os métodos utilizados para combinar as descobertas dos estudos foram adequados? 5 critérios; 10$)$ Foi avaliada a probabilidade de viés de publicação (também conhecido como efeito "gaveta de arquivo")? $\underline{3}$ critérios; e 11) O conflito de

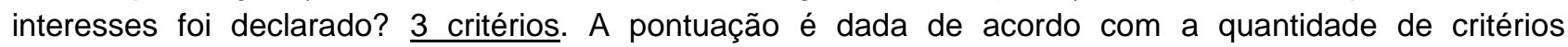
obedecidos pelo estudo selecionado para avaliação da seguinte maneira: 4 e 3 critérios $=4$ pontos; 3 e 2 critérios $=3$ pontos; 2 e 1 critério $=2$ pontos; 1 e 0 critérios $=1$ ponto. (KUNG J, et al., 2010).

Tabela 1 - Estudos avaliados pelo checklist R-AMSTAR.

\begin{tabular}{cccc}
\hline \multirow{2}{*}{ Itens } & \multicolumn{3}{c}{ Autor } \\
\cline { 2 - 4 } & Saquetto MB, et al., 2019 & Mehrholz J, et al., 2011 & Buzelli ARM, et al., 2014 \\
\hline Item 1 & 2 & 4 & 4 \\
Item 2 & 2 & 2 & 4 \\
Item 3 & 1 & 4 & 3 \\
Item 4 & 1 & 1 & 3 \\
Item 5 & 3 & 2 & 3 \\
Item 6 & 4 & 4 & 4 \\
Item 7 & 4 & 4 & 3 \\
Item 8 & 2 & 3 & 3 \\
Item 9 & 3 & 3 & 1 \\
Item 10 & 2 & 3 & 3 \\
Item 11 & 2 & 3 & 32 \\
\hline Total & 33 & 33 & Moderado \\
\hline Qualidade & Moderado & Moderado & \\
\hline
\end{tabular}

Fonte: Batista PBC, et al., 2020.

\section{RESULTADOS E DISCUSSÃO}

Como resultados da pesquisa nas bases de dados, foram encontrados 156 estudos a partir dos termos de busca utilizados. No entanto, 152 artigos foram excluídos, sendo 57 por títulos irrelevantes, 44 publicados fora do período especificado, 31 duplicados, 7 estudos pilotos, 3 estudos não publicados na íntegra, se enquadrando nos critérios pré estabelecidos apenas 4 dos 156 artigos encontrados. Dos 4 estudos selecionados, 3 eram revisões sistemáticas e 1 era ensaio clínico controlado randomizado (Quadro 1). 
Quadro 1 - Estudos selecionados para avaliação.

\begin{tabular}{|c|c|c|c|c|}
\hline Autor/Ano & Título & Objetivo do estudo & Intervenção & Resultados e Conclusão \\
\hline \multirow{2}{*}{$\begin{array}{l}\text { Saquetto MB, } \\
\text { et al., } \\
2019\end{array}$} & \multirow{2}{*}{$\begin{array}{l}\text { Exercícios aquáticos } \\
\text { no funcionamento e } \\
\text { na qualidade de vida } \\
\text { de pessoas pós- } \\
\text { AVC: uma revisão } \\
\text { sistemática e } \\
\text { metanálise }\end{array}$} & \multirow{2}{*}{$\begin{array}{l}\text { Investigar os efeitos } \\
\text { do exercício à base } \\
\text { de água no } \\
\text { funcionamento e na } \\
\text { qualidade de vida em } \\
\text { pessoas pós-AVC }\end{array}$} & \multirow{2}{*}{$\begin{array}{l}\text { A intervenção experimental foi } \\
\text { exercícios aquáticos que } \\
\text { consiste em protocolos de } \\
\text { exercícios contra a resistência à } \\
\text { água. Já a controle foram } \\
\text { exercícios terrestres. }\end{array}$} & $\begin{array}{l}\text { Resultado: a metanálise demonstrou melhora significativa nas limitações de } \\
\text { forma global, sendo física, emocional, mental, social e na dor corporal, para } \\
\text { participantes do grupo de exercícios à base de água. }\end{array}$ \\
\hline & & & & $\begin{array}{l}\text { Conclusão: O exercício na água pode ser uma alternativa para inclusão em } \\
\text { programas de reabilitação, visto que apresentou muitos benefícios aos } \\
\text { participantes desse grupo. }\end{array}$ \\
\hline \multirow{2}{*}{$\begin{array}{l}\text { Mehrholz J, et } \\
\text { al., } \\
2011\end{array}$} & \multirow{2}{*}{$\begin{array}{l}\text { Exercícios à base de } \\
\text { água para melhorar as } \\
\text { atividades da vida } \\
\text { diária após acidente } \\
\text { vascular cerebral: } \\
\text { revisão sistemática }\end{array}$} & \multirow{2}{*}{$\begin{array}{c}\text { Investigar o efeito de } \\
\text { exercícios à base de } \\
\text { água para reduzir a } \\
\text { incapacidade após um } \\
\text { acidente vascular } \\
\text { cerebral. }\end{array}$} & \multirow{2}{*}{$\begin{array}{c}\text { Exercícios à base de água como } \\
\text { qualquer intervenção individual ou } \\
\text { em grupo, incluindo apenas } \\
\text { estudos que consideram } \\
\text { tratamentos realizados por uma } \\
\text { equipe treinada. }\end{array}$} & $\begin{array}{l}\text { Resultado: Houve melhora significativa nas AVDs, capacidade funcional e na } \\
\text { força muscular, porém os resultados foram baseados em estudos únicos, não } \\
\text { havendo melhora significativa na capacidade de andar, equilíbrio postural ou } \\
\text { condicionamento físico. }\end{array}$ \\
\hline & & & & $\begin{array}{l}\text { Conclusão: As evidências de ensaios clínicos randomizados não confirmam } \\
\text { que exercícios à base de água ajudem a reduzir a incapacidade após } \\
\text { acidente vascular cerebral, faltando evidências. }\end{array}$ \\
\hline \multirow{2}{*}{$\begin{array}{l}\text { Lee ME, et al., } \\
2016\end{array}$} & \multirow{2}{*}{$\begin{array}{l}\text { Eficácia do treinamento } \\
\text { em esteira aquática na } \\
\text { simetria e equilíbrio da } \\
\text { marcha em pacientes } \\
\text { com AVC subagudo }\end{array}$} & \multirow{2}{*}{$\begin{array}{l}\text { Determinar a eficácia } \\
\text { do treinamento em } \\
\text { esteira aquática (ATT), } \\
\text { como uma nova } \\
\text { modalidade na } \\
\text { reabilitação de AVC }\end{array}$} & \multirow{2}{*}{$\begin{array}{c}\text { Realizou-se quinze sessões de } \\
\text { intervenção de ATT por três } \\
\text { semanas, as sessões foram } \\
\text { realizadas cinco vezes por } \\
\text { semana com duração de vinte } \\
\text { minutos, sendo quatro fases em } \\
\text { velocidades diferentes, a cinco } \\
\text { minutos de duração por fase. }\end{array}$} & $\begin{array}{l}\text { Resultado: Do pré ao pós intervenção foram observadas melhoras } \\
\text { estatisticamente significativas no CWT, BBS e ABC, a simetria do passo e a } \\
\text { simetria temporal geral demonstraram melhora sem significância estatística. }\end{array}$ \\
\hline & & & & $\begin{array}{l}\text { Conclusão: O ATT melhora os aspectos funcionais da marcha, incluindo } \\
\text { CWT, BBS, ABC e simetria espaço temporal da marcha, embora sem } \\
\text { significância estatística, mais estudos são necessários para examinar e } \\
\text { comparar os benefícios potenciais da TCA como uma nova modalidade para } \\
\text { a terapia do AVC, com outras modalidades. }\end{array}$ \\
\hline \multirow[t]{2}{*}{$\begin{array}{l}\text { Buzelli ARM, et } \\
\text { al., } \\
2014\end{array}$} & \multirow{2}{*}{$\begin{array}{c}\text { Os efeitos da terapia } \\
\text { aquática na mobilidade } \\
\text { de indivíduos com } \\
\text { doenças neurológicas: } \\
\text { uma revisão } \\
\text { sistemática }\end{array}$} & \multirow{2}{*}{$\begin{array}{l}\text { Resumir as evidências } \\
\text { sobre os efeitos da } \\
\text { terapia aquática na } \\
\text { modalidade de } \\
\text { indivíduos com } \\
\text { doenças neurológicas. }\end{array}$} & \multirow{2}{*}{$\begin{array}{c}\text { Foi definida a terapia aquática } \\
\text { como um programa, usando } \\
\text { características mecânicas e } \\
\text { térmicas da água durante parcial } \\
\text { ou completa imersão como um } \\
\text { tratamento individual ou em grupo, } \\
\text { incluindo qualquer método de } \\
\text { tratamento, movimento, exercício } \\
\text { ou atividade em uma piscina }\end{array}$} & $\begin{array}{l}\text { Resultado: Um total de cento e dezesseis artigos foram obtidos para a } \\
\text { elegibilidade do texto completo, onde vinte estudos atenderam a inclusão } \\
\text { específica, onde são quatro ensaios clínicos randomizados, quatro estudos } \\
\text { não randomizados e doze testes antes e depois, demonstram evidências } \\
\text { "justas" na melhora da velocidade da marcha após a terapia aquática. }\end{array}$ \\
\hline & & & & $\begin{array}{l}\text { Conclusão: A síntese demonstrou evidências que apoiam o uso da terapia } \\
\text { aquática para melhorar o equilíbrio dinâmico e a velocidade da marcha em } \\
\text { adultos com certas condições neurológicas. }\end{array}$ \\
\hline
\end{tabular}

Fonte: Batista PBC, et al., 2020. 
É evidente que o tratamento realizado com hidroterapia apresenta resultados benéficos a pacientes que tiveram AVC. Os efeitos deste recurso se mostram eficazes no tratamento das sequelas recorrente do AVC, principalmente no espasmo muscular, pois oferece uma técnica cientificamente inovadora que apresenta resultados satisfatório na reabilitação dos pacientes (SAQUETTO MB, et al., 2019).

Os sinais e sintomas clínicos que os pacientes com essas sequelas irão apresentar são: algias, posturas anormais, contraturas, deformidades, movimentos involuntários (espasticidade), astenia, atrofia muscular, reflexos tendinosos profundos, sinal de Babinski, movimentação seletiva lenta, diminuição/perda de habilidade dos movimentos e alteração da elasticidade da fibra muscular. A musculatura afetada em membros superiores são: retratores da escápula; adutores, depressores e rotadores internos do ombro; flexores do cotovelo; pronadores do antebraço; flexores de punho e dedos, já em membros inferiores são: os retratores pélvicos; adutores, rotadores internos e extensão do quadril; extensores do joelho; flexores plantares; supinadores e flexores dos dedos. (FELICE TD, et al., 2011; BASTOS VPD, et al., 2016).

A espasticidade é o distúrbio mais comum do neurônio motor superior sendo ele caracterizado pela excitação excessiva dos motoneurônios responsáveis pela inervação dos músculos que estimulam o tônus muscular, resultando em uma desinibição que leva a um aumento da atividade dos neurônios alfa e gama provocando uma contração muscular ativa, caracterizando-se por sinais inadequados, que em vez de relaxar a musculatura ocorre uma contração rápida e irregular, fazendo com que o movimento muscular se exceda e haja um estiramento das fibras musculares que sensibilizará o fuso ativo dos motoneurônios, gerando assim um encurtamento muscular e acarretando com que o movimento ocorra de forma acelerada, sendo uma contração concêntrica. O lado afetado ficará flácido, tendo este estado apenas horas ou no máximo alguns dias, após essa flacidez se instalará a espasticidade. Acredita-se que $40 \%$ das pessoas acometidas pelo AVC desenvolvem a espasticidade, portanto a mesma será um fator incapacitante no seu dia a dia, tornando-os dependentes dos familiares (ARAUJO APS, et al., 2012; MORO CHC, 2012).

A partir da incapacidade funcional que o paciente venha desenvolver é de muita importância que ele realize tratamento fisioterapêutico para que os déficits não venham a progredir, vale ressaltar que além da fisioterapia em solo, a hidroterapia é um dos recursos utilizados na reabilitação de pacientes com disfunções do sistema nervoso, pois utiliza as propriedades físicas da água, a hidroterapia tem bases biomecânicas e termodinâmicas possibilitando ao paciente alcançar facilmente metas e desafios que por sua vez em solo seja impossível realizar (MIRANDA MR, et al., 2018).

O estudo de Zanatto D, et al. (2013), afirma que pacientes com sequelas de AVC, apresentam melhora de mobilidade após tratamento com hidroterapia. Foi realizado a inclusão de 9 pacientes com paraparestesia espástica hereditária (HSP), com o objetivo de avaliar a melhora da marcha nesses pacientes, com um programa de hidroterapia de 10 semanas, sendo 2 vezes na semana com cada sessão de 45 minutos, cada participante foi submetido a um método de avaliação, sendo utilizado a Escala de Ashwoth Modificada para identificar o nível de espasticidade muscular e uma análise biomecânica no início e no final do tratamento. Ao fim do tratamento proposto, pode ser observado um aumento da força muscular e flexibilidade nas articulações, a parti destes resultados tendo o aumento da velocidade de caminhada e comprimento de passo. Os mesmos destacam o potencial para terapia que visam na melhora ou aumento da amplitude de movimento e força para ter um efeito benéfico na velocidade da marcha do paciente.

Saquetto MB, et al. (2019), realizou uma revisão sistemática, onde foi incluído em sua metanálise 15 estudos, com um número que variou de 12 a 120 pacientes, com idades entre 44 a 77 anos, incluíam no tratamento aquático exercícios aeróbicos e de força, a duração dos programas de tratamento variava de 2 a 12 semanas, sendo realizados de 2 a 6 vezes na semana, com sessões de 30 a 60 minutos, nesta pesquisa foi realizado comparações nos protocolos de tratamento onde uns eram combinados com terapia sem solo e outros separados, a partir dos resultados encontrados, os autores concluíram que a hidroterapia combinada com a terapia em solo apresenta resultados satisfatório, pois o método é muito eficaz na melhora da força muscular, equilíbrio, mobilidade articular, na capacidade aeróbica, na velocidade a marcha e no alcance funcional. Porém no estudo de Mehrholtz J, et al. (2011), selecionou 4 artigos em sua pesquisa e com o resultado, teve a conclusão que os pacientes obtiveram uma melhora nas atividades de vida diária e na 
força muscular, ainda assim mesmo com os resultados ele conclui que ainda são fracas as evidencias quanto ao benefício da hidroterapia quando aplicado na melhora da capacidade funcional em pacientes com sequelas neurológicas, indica que são necessários estudos futuros, pois ainda há evidencias insuficientes devido ao pequeno número de ensaios clínicos randomizados.

Lee ME, et al. (2017), afirma que o tratamento com hidroterapia é eficaz associado a outros métodos, onde realizou um estudo com um protocolo de tratamento de hidroterapia com 21 pacientes que sofrearam um AVC, utilizando uma esteira aquática, durante 7 semanas, com 15 sessões que eram realizadas 5 vezes na semana, sendo estabelecido 20 minutos cada sessão, a velocidade da esteira mudava a cada 5 minutos, os pacientes tinham um intervalo de 2 minutos, neste protocolo incluía alongamentos, fortalecimento e treino de marcha, ao fim do tratamento ser observado uma melhora na velocidade da marcha, na assimetria da marcha, equilíbrio, o presente estudo pode determinar a eficácia do tratamento na hidroterapia utilizando o treino de esteira aquática na reabilitação de pacientes com sequelas de AVC.

Os estudos demostraram que a hidroterapia, como recurso fisioterapêutico para tratar sequelas neurológicas é eficaz, pois em todos eles, os resultados foram satisfatórios, mostrando que através das propriedades da água, tem sido um tratamento benéfico para pacientes com tal patologia.

\section{CONSIDERAÇÕES FINAIS}

A hidroterapia oferece resultados satisfatórios quando combinados ou não com terapia em solo e/ou com junção de outros métodos de tratamento em pacientes com sequelas de AVC tornando-se um recurso eficaz na melhora do equilíbrio, no ganho de força muscular, no controle da espasticidade, na amplitude de movimento e na mobilidade dos pacientes, fazendo com que eles tenham uma melhora em sua funcionalidade e da qualidade de vida desses indivíduos, já que essas sequelas tendem a regredir com o tratamento, possibilitando que eles tenham um retorno agradável em suas atividades de vida diária. No entanto, a hidroterapia ainda é um recurso complementar da terapia em solo.

\section{REFERÊNCIAS}

1. ARAÚJO APS, et al. Prevalência dos fatores de risco em pacientes com acidente vascular encefálico atendidos no setor de neurologia da clínica de fisioterapia da UNIPAR. Arq. Ciênc. Saúde Unipar. 2008; 12(1): 35-42p.

2. BASTOS VPD, et al. Benefícios da Hidroterapia nos Pacientes Portadores de Sequela de Acidente Vascular Cerebral: uma revisão de literatura. Saúde (santa maria), Fortaleza - CE, jul. 2016.

3. BENSENOR IM, et al. Prevalência de acidente vascular cerebral e de incapacidade associada no Brasil: Pesquisa Nacional de Saúde - 2013. Arquivo de Neuropsiquiatria. 2010, 73(9):746-750.

4. BRASIL. MINISTÉRIO DA SAÚDE. Secretaria de Atenção à Saúde. Departamento de Ações Programáticas Estratégicas. Acidente vascular cerebral (AVC) [Internet]. BVMS. 2015 [cited 2020 Mar 20]. Disponível em: http://bvsms.saude.gov.br/dicas-em-saude/2188-avc-acidente-vascular-cerebral, acessado em: 14 abr. 2020.

5. BRUCE E, BECKER AJC. Terapia Aquática Moderna. $1^{\circ}$ ed. São Paulo: Manole; 2000. 188 p.

6. BUZELLI ARM, et al. The effects of aquatic therapy on mobility of individuals with neurological diseases: a systematic review. Clinical Rehabilitation. 2014; 29(8): 741-751p.

7. CHÃO CC, et al. Fisioterapia aquática nas disfunções do aparelho locomotor. 2004.

8. DONATO H, DONATO M. "Stages for undertaking a systematic review", Acta Medica Portuguesa. 2019; 32(3): 227235p.

9. FELICE TD, et al. Eletroestimulação e Crioterapia para espasticidade em pacientes acometidos por Acidente Vascular Cerebral. Rev. Neurociências. Dourados-MS. 2011; 1(19): 77-84p,

10. FINHOLDT MC. "Análise da função autonômica sobre o sistema cardiovascular em humanos submetidos à mudança postural e imersão em água". Tese de Mestrado em Patologia Clínica. Universidade Federal do Triângulo Mineiro, UFTM, Brasil. 2007.

11. GIMENES RO, et al. Análise crítica de ensaios clínicos aleatórios sobre fisioterapia aquática para pacientes neurológicos. Rev Neurociências. 2019; 13(1): 5-10.

12. KUNG J, et al. From Systematic Reviews to Clinical Recommendations for Evidence- Based Health Care: Validation of Revised Assessment of Multiple System- atic Reviews (R-AMSTAR) for Grading of Clinical Relevance. 84 Open Dent J. 2010; 4: 84-91. 
13. LEE ME, et al. Efficacy of aquatic treadmill training on gait symmetry and balance in subacute stroke patients. Ann Rehabil Med. 2017; 41(3): 376-386.

14. LIANZA S, et al. Diagnóstico e Tratamento da Espasticidade. Soc Bras Med Física e Reabil. 2001; 1-12.

15. LOCATELLI MC, et al. Perfil epidemiológico dos pacientes com acidente vascular cerebral isquêmico atendidos em um hospital Epidemiological. profile of patients with ischemic stroke treated in a Hospital. Artig Orig Rev Soc Bras Clin Med. 2017;15(3):150-154.

16. MEHRHOLZ J, et al. Water-based exercises for improving activities of daily living after stroke. Stroke. 2011; 42(4).

17. MIRANDA MR, et al. Benefícios Da Hidroterapia Em Pacientes Após Acidente Vascular Celebral (AVC). Benefits of Hydrotherapy in Patients After Celebral Vascular Accident (Avc). Rev Iniciação Científica e Extensão. 2018; 1(5): 465-471.

18. MORO CHC. O que é espasticidade pós AVC. 2012.

19. O'SULLIVAN SB, THOMAS J, SCHMITZ GDF. Fisioterapia: Avaliação e Tratamento. $5^{\circ}$ ed. São Paulo: Manole; 2010. 1506p.

20. PIASSAROLI CAP, et al. Modelos de reabilitação fisioterápica em pacientes adultos com sequelas de AVC isquêmico. Rev Neurociencias. 2012; 20(1): 128-137.

21. ROUTI RG, MORRIS DM. Reabilitação Aquática. $1^{\circ}$ ed. São Paulo: Manole; 1997. 463p.

22. ROWLAND LP, JUNIOR HHM, ARAÚJO CLC. Merritt Tratado de Neurologia. $10^{\circ}$ Rio de Janeiro: Guanabara Koogan; 2002. 884p.

23. SAQUETTO MB, et al. Water-Based Exercise on Functioning and Quality of Life in Poststroke Persons: A Systematic Review and Meta-Analysis. J Stroke Cerebrovasc Dis. 2019; 28(11).

24. SPRINGHOUSE ACC. Atlas de Fisiopatologia. $1^{\circ}$ ed. Rio de Janeiro: Guanabara Koogan; 2004, 430p.

25. ZANATTO D, et al. Hemiplegia : uma revisão bibliográfica. III Encontro Ciêntífico do GEPro, Grup Estud Produção [Internet]. 2013; disponível em: http://geprofatecjahu.com.br/anais/2013/24.pdf. Acessado em: 20 abr 2020. 